\title{
Virtual indoor-GPS for measurement uncertainty determination in reconfigurable environments
}

\author{
Christoph Nicksch ${ }^{1} \mathbb{D} \cdot$ Mielad Sabzehi ${ }^{1} \cdot$ Robert H. Schmitt $^{2}$
}

Received: 30 November 2021 / Accepted: 31 January 2022 / Published online: 10 February 2022

(c) The Author(s) 2022

\begin{abstract}
In Line-less Mobile Assembly Systems, the mobilization of assembly resources and products enables rapid physical system reconfigurations to increase flexibility and adaptability. The clean floor approach discards fixed anchor points, so that assembly resources such as mobile robots and automated guided vehicles transporting products can adapt to new products and form new processes. Associated challenges are accurate spatial referencing between mobile resources to meet assembly tolerance requirements. There is a need for more accurate positioning data to locate and navigate mobile assembly resources. An indoor-GPS, as a distributed large-scale metrology system, is able to cover a wide shop floor area and to obtain positioning data with uncertainties in the submillimeter range. The measurement uncertainty of such a system depends on the spatial distribution of the transmitters and the receiver positions. To be able to validate positioning tolerance requirements of an assembly process, measurement uncertainties must be determined. Virtual measurements simulate measurement processes and model dependencies between the environment and the metrology system. This work presents a novel approach for a virtual indoor-GPS to determine measurement uncertainties during a process and to evaluate the measurement process capability. Experiments show the validity of the virtual indoor-GPS which can be used as a planning tool for metrology system setups within Line-less Mobile Assembly Systems.
\end{abstract}

Keywords Measurement uncertainty $\cdot$ Distributed large-scale metrology $\cdot$ Indoor-GPS $\cdot$ Line-less mobile assembly systems

\section{Introduction}

The assembly of large-scale products is characterized by a high level of effort in terms of logistics and the set-up processes [5]. This results in a high depth of added value, which in turn leads to the requirement to produce large components "First-Time-Right" e.g. by applying the concept

Christoph Nicksch

c.nicksch@wzl.rwth-aachen.de

Mielad Sabzehi

mielad.sabzehi@rwth-aachen.de

Robert H. Schmitt

r.schmitt@wzl.rwth-aachen.de

$1 \quad$ Laboratory for Machine Tools and Production Engineering WZL, RWTH Aachen University, Campus-Boulevard 30, 52074 Aachen, Germany

2 Laboratory for Machine Tools and Production Engineering WZL, Fraunhofer Institute for Production Technology IPT, RWTH Aachen University, Campus-Boulevard 30, 52074 Aachen, Germany of measurement assisted assembly [12, 18, 29]. Schmitt [41] defines the term large-scale product by the relatively small ratio of tolerance and nominal dimension, leading to complex challenges and problems in the production. Industrial use cases (e.g. airplane, energy systems and maritime engines industry) show that for example tolerances of $1 \mathrm{~mm}$ are common for nominal dimensions of up to $30 \mathrm{~m} \mathrm{[31].} \mathrm{The}$ assembly of large-scale products is also affected by fluctuating demands and increasing number of variants, which enforces the wish of more flexible assembly systems. The concept of Line-less Mobile Assembly Systems (LMAS) can address new product variants by rapidly reconfiguring assembly stations by integrating mobile resources such as mobilized assembly robots and automated guided vehicles [19]. LMAS discards fixed shop floor layouts and follows a so-called clean floor approach, which provides a sizeable, coherent shop floor space. Since there are less fixed anchor points for localization and navigation of assembly resources, factory-wide positioning data of assembly resources become relevant. [11, 12, 27, 40]. Therefore, distributed metrology systems must provide a coordinate system for spatial 
referencing on the entire shop floor [7]. Following this idea, Demeester [8] defined a Global Reference System (GRS) as a system covering the measuring volume of an entire shop floor and enabling measurements at many points simultaneously. Implementing a GRS requires a distributed metrology system that is scalable across the assembly environment and can simultaneously measure multiple objects in all six degrees of freedom.

Nicksch et al. [31] reviewed current large-scale metrology (LSM) systems to be used within a GRS. Based on criteria from airplane manufacturing as well as the mentioned GRS criteria, a laser tracker, a laser radar and an indoor-GPS (iGPS) system are compared, with the conclusion that only the iGPS fulfills all requirements for a GRS implementation. Unique characteristics of the iGPS [4] are a scalable measuring volume and the ability of measuring 6D-poses of several objects simultaneously. To evaluate the suitability in detail, the measurement uncertainty for the respective processes has to be considered. The iGPS measurement uncertainty depends on the spatial distribution of transmitters (called configuration in this work) which affects minimum tolerances to be checked and accordingly the measurement process capability [10].

Facing challenges of LSM systems within the mentioned environments, there are three crucial requirements which can be identified: First, the metrology system must be reconfigurable to be able to adapt to changing assembly environments. Second, the metrology system must be able to simultaneously measure several mobile resources as well as products with uncertainties in the submillimeter range covering the entire shop floor. Third, the measurement uncertainty must be determined in advance to ensure that narrow assembly tolerances can be checked when the assembly system is changed.

The aim of this work is therefore to introduce a virtual iGPS as a planning tool to enable measurement uncertainty determination depending on the iGPS configuration and the assembly environment. In the following (see Sect. 2), we briefly summarize the functional principle and the usage purpose of the iGPS within reconfigurable environments such as LMAS. Afterwards, we review the state of the art regarding existing approaches for iGPS measurement uncertainty determination to derive requirements for the development of the proposed virtual iGPS. The next sections follow the structure provided by the guide to the expression of uncertainty in measurements (GUM) [21] which comprises the identification of relevant impact factors, modelling the measurement process and deriving the measurement uncertainty model. Accordingly, Sect. 3 deals with the development and validation of the virtual iGPS as a measurement model. In Sect. 4, the virtual iGPS is used to determine the measurement uncertainty. A validation is carried out in Sect. 5 by comparing model output with literature knowledge and by simulating iGPS configurations for an assembly use case from airplane manufacturing.

\section{State of the art: distributed large-scale metrology in assembly}

This section creates the knowledge base to understand the subsequent sections. It comprises the iGPS as a suitable metrology system obtaining factory-wide positioning data and virtual measurements as a planning tool for assembly. Furthermore, it contains existing approaches to model iGPS measurements deriving deficits as a motivation for this work.

\section{1 iGPS as a suitable and complex reference system}

As elaborated in Sect. 1, the iGPS is a suitable measurement system for reconfigurable assembly of large-scale products. To understand the particular challenge of modeling iGPS measurements, its functional principle and impact factors on uncertainty are briefly introduced in the following section.

The iGPS is a scalable system consisting of arbitrary number of transmitters and receivers [14]. The location of transmitters has to be determined before starting measurements by a calibration routine. Each transmitter provides two measurement values to each receiver: the horizontal (azimuth, $\varphi$ ) and the vertical (elevation, $\theta$ ) angles. Receivers can calculate their position whenever they are localized in the line of sight (LOS) of three or more transmitters. Each transmitter generates two rotating infrared laser beams and an infrared LED strobe with a defined rotation frequency. These optical signals are converted into timing pulses, which are used for angle calculation of $\varphi$ and $\theta$. Triangulation is used to determine the receiver's position. The underlying patent [16] provides a more detailed description of the calculation of angle values based on timing pulses. Figure 1 illustrates an iGPS configuration comprising three transmitters and a receiver, where $d$ symbolizes the measurement distance between a transmitter and a receiver.

A technically feasible iGPS configuration depends on the working range, which is defined by: a permitted distance between a transmitter and a receiver $(2-30 \mathrm{~m})$, a minimum distance between transmitters $(2 \mathrm{~m})$, a minimum distance between transmitters and receivers $(2 \mathrm{~m})$ and a permitted elevation angle between a transmitter and a receiver (+/- 30ř). The configuration and the calibration are the impact factors with the highest magnitude on the measurement uncertainty that can be manipulated by the operator. The measurement uncertainty contribution of the configuration can be up to $18 \%$ and that of the calibration up to $27 \%$ of the total measurement uncertainty budget [34]. An inhomogeneous uncertainty distribution with different magnitudes over the 
Fig. 1 iGPS configuration with three transmitters and a receiver

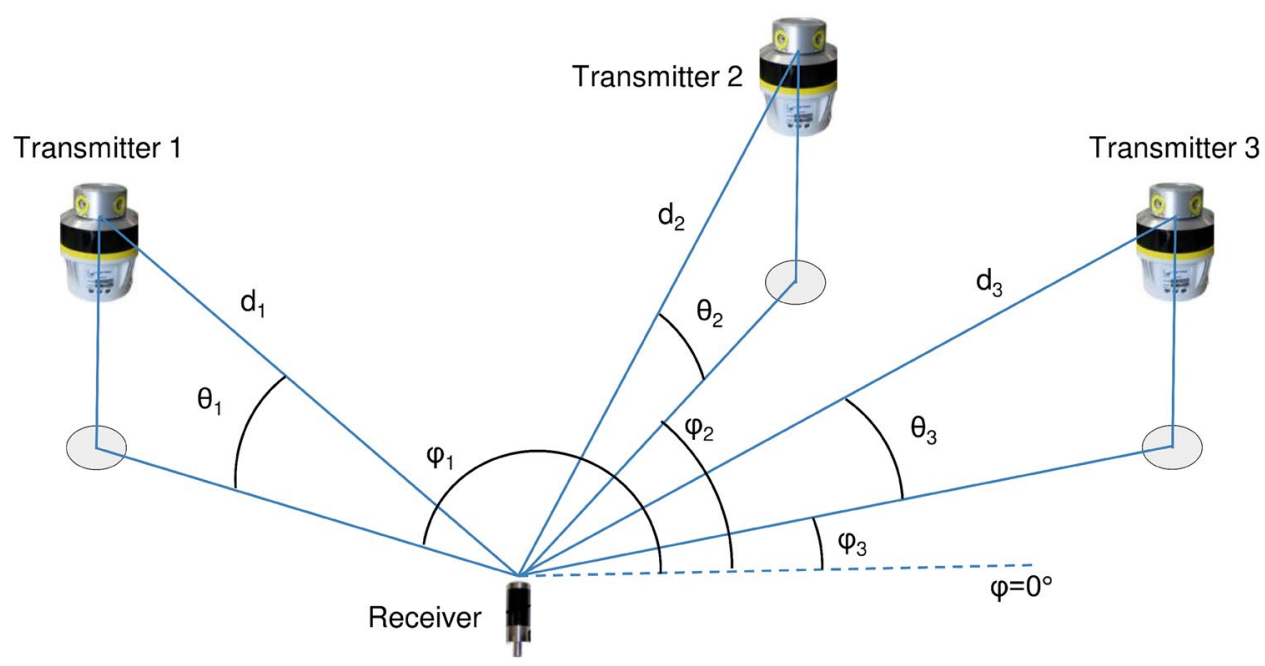

measurement volume was detected in [9, 17, 34, 38, 43], which characterizes a complex measurement process according to the guideline VDI/VDE 2600-2 [45].

\subsection{Related works}

To operate distributed LSM systems such as the iGPS economically, Franchescini et al. [14] postulate an inherent intelligence to continuously and automatically adapt distributed LSM systems to new requirements of measurement objects. Leach et al. [24] introduce the approach of "information-rich metrology" for this purpose. In addition to information about the measurement object, other information sources, e.g. the physical measurement principle and the assembly environment as well as historical data of the measurement object, are considered to increase the quality of the measurement. The authors define increased quality as a reduction of measurement time and uncertainty, as well as a more demand-oriented coverage of the measurement volume. The different sources of information are used as input variables for model building of virtual measurements to optimize the measurement planning.

Similar to Leach's idea, Schmitt [42] defines the term virtual measurement (or "measuring on the model"). This means that, independent of the measurement task, a digital model of the component is first created and digitally preserved. Individual features can then be subsequently measured and evaluated on this model, enabling a priori determination of the measurement uncertainty. This forms the basis for an optimization of the measurement planning considering assembly tolerance requirements.

Following this idea, Quinders [34] develops a "virtual prototype" as a planning tool for measurement-assisted assembly processes and also focuses on distributed LSM. Figure 2 shows the concept of the virtual prototype, which forms the framework for this work. The concept is used within reconfigurable assembly systems after the product, required process flows, trajectories of assembly resources have been defined: The virtual measurement provides the necessary data for the measurement uncertainty determination. Comparing resulting uncertainties with required

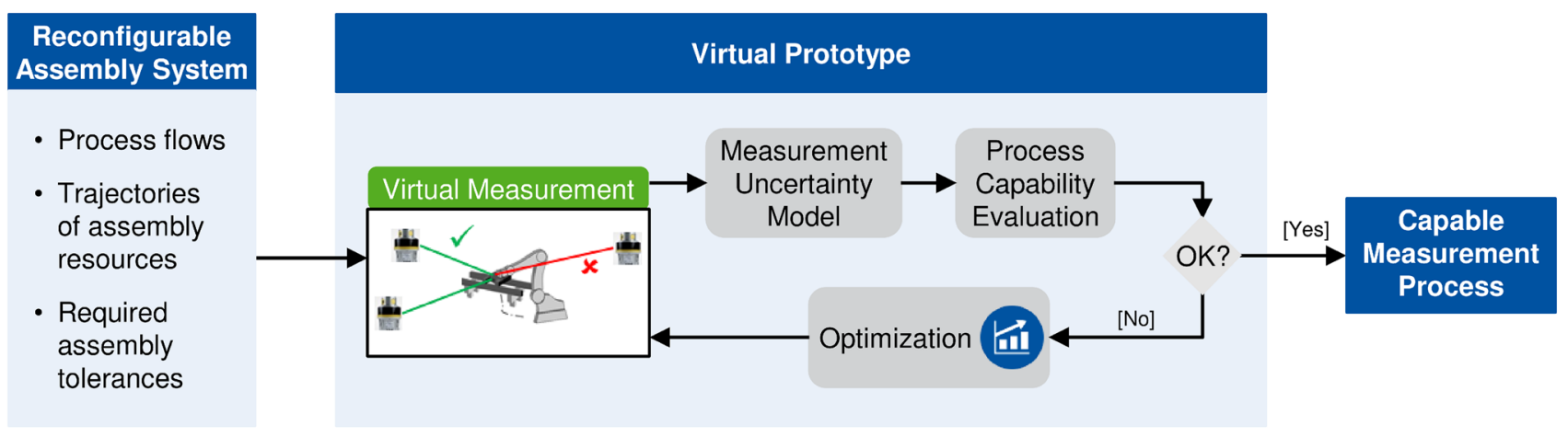

Fig. 2 Virtual measurements within optimization of reconfigurable assembly systems 
assembly tolerances enables an estimation of the process capability [39]. If the determined process capability value meets the requirements, a feasible configuration is found enabling capable measurement processes, whereas an insufficient value leads to another optimization cycle. Quinders [34] summarizes that the virtual prototype decreases both the setup times of measurement-assisted assembly processes and the measurement uncertainty, which enables capable measurements for processes with narrower tolerances.

\subsection{Modelling complex measurement processes}

The GUM [21] is the scientific standard for determining measurement uncertainty. Thus, it can also be applied to complex measurement processes, but it does not provide any assistance in modelling and validating the dependency between the influencing factors and the measurand defining the measurement process. Therefore, Müller et al. [30] propose artificial neural networks for modelling complex measurement processes, reducing time effort and the need for expert knowledge. Still, the approach has not been applied to complex measurements yet for validation purposes.

Forbes [13] develops a reference model for multi-station systems (such as the iGPS consisting of several transmitters) to estimate target positions. He uses numerical optimization to determine model parameters for target localization. As a disadvantage, he summarizes that real measurements must be carried out in advance, which in turn does not allow using this approach for simulation purposes within the virtual measurement concept.

Apart from these theoretical approaches, various publications deal in particular with measurement uncertainty models of the iGPS. Hughes et al. [17] investigate the impact of inter-target distance on the iGPS measurement uncertainty using a setup of 12 transmitters and a laser tracker as reference system. The validation shows that the uncertainty can be up to $0.375 \mathrm{~mm}$ and that a simple linear model cannot be used to describe the iGPS uncertainty assuming a more complex system behavior.

Sun et al. [43] develop an analytical model of iGPS measurements based on triangulation of transmitter pairs that can be arbitrarily extended by additional transmitters. Simulated target positions and given transmitter positions form the inputs of the model. The model is used to derive an uncertainty model according to GUM [21] considering transmitter rotation uncertainties as the only impact factor. A comparison with a laser tracker emphasizes that there are still significant differences of about $50 \%$ between observed and expected values of the uncertainty.

Guo et al. [15] propose a measurement process model for a so-called workshop Measurement Positioning System (wMPS) which is similar to the functional principle of the iGPS. By simulating different transmitter configurations with the model and conducting experiments, it was qualitatively confirmed that there is a correlation between an increasing number and density of transmitters and a decreasing value of the measurement uncertainty. Still, there is no statistical validation of the model and relevant impact factors such as the calibration are not considered.

Based on the Patent [16] Jingtao et al. [20] develop an iGPS measurement process model and use it to optimize transmitter layouts. They show that by using the measurement model as an objective function, the measurement uncertainty can be reduced from 0.3 to $0,14 \mathrm{~mm}$. Even though they demonstrate that the optimization converges, there is no validation of the measurement model and the effects of calibration are also not considered.

Quinders [34] investigates the effects of different iGPS transmitter configurations on the measurement uncertainty using a calibrated inter-target distance. The uncertainty model comprises the most relevant impact factors known in literature (such as calibration, number of LOS and configuration). Due to the constant inter-target-distance, the model cannot be used for arbitrary positioning of iGPS receivers to determine uncertainties for absolute coordinates, as it is required in industrial assembly.

The presented approaches emphasize that there is currently no validated iGPS measurement process model which can be used for uncertainty determination of absolute coordinates for the establishment of a GRS within assembly. Either the existing models do not cover all relevant impact factors or only small inter-target-distances are considered, which cannot be transferred to the measurement distances in large-scale product assembly. Still, theoretical knowledge for modelling complex measurement processes exists, provided by [13,21,30]. This work follows the idea of [13] to apply numerical optimization to consider redundant position information based on angle measurements from several transmitters (see Fig. 1), since mathematically only two transmitters are required for triangulation, as shown in [43]. The measurement process model is called virtual iGPS in this work and forms the basis, on the one hand, to derive the measurement uncertainty model according to GUM [21] and on the other hand, to enable virtual measurements for process capability determination in assembly, as illustrated in Fig. 2.

\section{Virtual iGPS for variable configurations}

Following the GUM [21], this section introduces relevant impact factors on the measurement uncertainty of the iGPS based on literature knowledge. The measurement process is modelled in respect to the relevant impact factors. 


\subsection{Relevant impact factors}

The review of current literature shows that the iGPS configuration (see configuration parameters in Fig. 1 have a significant impact on the measurement uncertainty $[15,17$, $20,43]$ and is considered as a relevant impact factor in this work. Since the functional principle of the iGPS relies on triangulation which requires spatial reference points, the calibration determining transmitter coordinates as spatial reference points is considered as an additional impact factor. Quinders [34] confirms this assumption in his analysis of iGPS impact factors, where the measurement uncertainty budget of the calibration is up to $22 \%$ (see Fig. 3). As Fig. 3 shows, the impact of systematic errors are even larger (36\%). To analyze systematic errors, an external reference system is required. Assuming the measurement uncertainties of $0.2 \mathrm{~mm}$ (as given in [20, 43]), a reference system with a measurement uncertainty of $0.02 \mathrm{~mm}$ would be required following the "Golden rule of metrology" [33]. Considering measurement distances in large-scale product assembly greater than $10 \mathrm{~m}$, a suitable reference system that could meet these requirements is not known to the authors. For this reason, systematic errors are not considered in this work.

\subsection{Modelling iGPS measurements}

In order to model the measurement process, the input and output values of the model are defined first. In conformity to the measurement system and the general idea of obtaining position data, the output values of the model are defined by the Cartesian coordinates of an iGPS receiver. The input values are chosen accordingly to the impact factors introduced in Sect. 3.1 and to the available measurands provided by the iGPS. The calibration forms the first model input, providing the Euler poses of all transmitters in the iGPS world coordinate system. The configuration of the iGPS system can be expressed by the LOS of each transmitter (as shown in Fig. 1) and forms the second model input. Considering these input variables, the LOS vector $\mathbf{r}_{T_{i}}$ of each transmitter $i$, represented in transmitter specific coordinates, and the Euler pose $\mathbf{e}_{i}$ of each transmitter (with regard to the iGPS world coordinate system) form the inputs of the measurement model. To obtain output values in the desired iGPS world coordinate system, $\mathbf{r}_{T_{i}}$ must be transformed into the iGPS world coordinate system, which is represented by the normalized LOS vector $\mathbf{r}_{i}$. The transformation is given by $\mathbf{e}_{i}$ containing $\mathbf{t}_{i}$ as the translation of a transmitter with regard to the iGPS world coordinate system. The model assumes that the input variables are subject to independent, normally distributed random effects. For clarity, the variables are listed in Table 1.

Figure 2 illustrates an ideal iGPS system with the LOS intersecting in one point. The real measurement process is subject to uncertainties, leading to skew LOS which do not intersect. The Cartesian coordinates of an iGPS receiver $\mathbf{p}_{\text {rec }}$ represent the measurement value of an iGPS receiver obtained from real measurements. Given vector $\mathbf{t}_{i}$ as the position vector of transmitter $i$, vector $\mathbf{r}_{i}$ as the normalized LOS vector of transmitter $i$, then point $\mathbf{p}_{\min }$ describes the point of minimum Euclidean distance to all LOS. It is assumed that $\mathbf{p}_{\min }$ is close to the measurand $\mathbf{p}_{\text {rec }}$ and therefore that $\mathbf{p}_{\min } \approx \mathbf{p}_{\text {rec }}$ (see Sect. 4.2 for confirmation of the assumption). The squared Euclidean distance between a LOS vector and an arbitrary point $\mathbf{p}$ may be described as:
Fig. 3 Relevant impact factors on the iGPS measurement uncertainty according to Quinders [34] and considered factors in this work

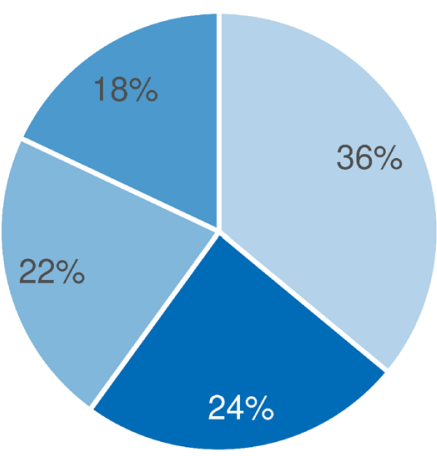

- Systematic error

- Random error

- Calibration

- Configuration considered impact factors

Table 1 Overview of variables

\begin{tabular}{ll}
\hline Variable & Description \\
\hline $\mathbf{r}_{T_{i}}$ & LOS vector of transmitter $i$ in transmitter specific coordinates \\
$\mathbf{r}_{i}$ & Normalized LOS vector of transmitter $i$ in iGPS world coordinates \\
$\mathbf{e}_{i}$ & Euler pose of transmitter $i$ in iGPS world coordinates \\
$\mathbf{t}_{i}$ & Position vector of transmitter $i$ in iGPS world coordinates \\
\hline
\end{tabular}


$d_{i}^{2}=\left|\left(\mathbf{p}-\mathbf{t}_{i}\right) \times \mathbf{r}_{i}\right|^{2}$

With the use of Lagrange's identity $d_{i}^{2}$ may be formulated as:

$d_{i}^{2}=\left|\mathbf{p}-\mathbf{t}_{i}\right|^{2}-\left|\left(\mathbf{p}-\mathbf{t}_{i}\right) \cdot \mathbf{r}_{i}\right|^{2}$

The sum of $N$ squared Euclidean distances may be denoted as $D$ :

$D=\sum_{i=1}^{N} d_{i}^{2}$

$D$ needs to be minimized in order to find $\mathbf{p}_{\text {min }}$ :

$\mathbf{p}_{\min }=\underset{\mathbf{p}}{\arg \min } D$

Following for a partial derivative with respect to $\mathbf{p}$ :

$\frac{\partial D}{\partial \mathbf{p}}=\sum_{i=1}^{N} \frac{\partial d_{i}^{2}}{\partial \mathbf{p}}=\sum_{i=1}^{N} 2\left(\mathbf{p}-\mathbf{t}_{i}\right)-2 \mathbf{r}_{i}\left[\left(\mathbf{p}-\mathbf{t}_{i}\right) \cdot \mathbf{r}_{i}\right]$

In order to minimize $D$ the partial derivative should equal to 0 :

$0=\sum_{i=1}^{N}\left(\mathbf{p}_{\min }-\mathbf{t}_{i}\right)-\mathbf{r}_{i}\left[\left(\mathbf{p}_{\min }-\mathbf{t}_{i}\right) \cdot \mathbf{r}_{i}\right]$

$\sum_{i=1}^{N} \mathbf{p}_{\min }-\mathbf{r}_{i}\left(\mathbf{p}_{\min } \cdot \mathbf{r}_{i}\right)=\sum_{i=1}^{N} \mathbf{t}_{i}-\mathbf{r}_{i}\left(\mathbf{t}_{i} \cdot \mathbf{r}_{i}\right)$

The following parts of Equation 7 may then be reformulated as a dyadic product:

$\mathbf{r}_{i}\left(\mathbf{p}_{\min } \cdot \mathbf{r}_{i}\right)=\left(\mathbf{r}_{i} \otimes \mathbf{r}_{i}^{T}\right) \mathbf{p}_{\min }$

$\mathbf{r}_{i}\left(\mathbf{t}_{\mathbf{i}} \cdot \mathbf{r}_{i}\right)=\left(\mathbf{r}_{i} \otimes \mathbf{r}_{i}^{T}\right) \mathbf{t}_{i}$

By factoring out $\mathbf{p}_{\min }$ and $\mathbf{t}_{i}$ :

$\sum_{i=1}^{N}\left(I-\mathbf{r}_{i} \otimes \mathbf{r}_{i}^{T}\right) \mathbf{p}_{\min }=\sum_{i=1}^{N}\left(I-\mathbf{r}_{i} \otimes \mathbf{r}_{i}^{T}\right) \mathbf{t}_{i}$

This equation may also be written as:

$A \mathbf{p}_{\text {min }}=b$

With:

$$
\begin{aligned}
& A=\sum_{i=1}^{N}\left(I-\mathbf{r}_{i} \otimes \mathbf{r}_{i}^{T}\right) \\
& b=\sum_{i=1}^{N}\left(I-\mathbf{r}_{i} \otimes \mathbf{r}_{i}^{T}\right) \mathbf{t}_{i}
\end{aligned}
$$

The equation can be solved numerically in respect to $\mathbf{p}_{\min }$ using a least-square-algorithm. With the assumption of $A$ and $(A \mid b)$ being matrices of full rank, the linear system of equations has a unique solution according to the RouchéCapelli theorem [36]. The virtual iGPS can be summarized for a number of $i$ transmitters:

$\mathbf{p}_{\min }=f\left(\mathbf{r}_{T_{i}}, \mathbf{e}_{i}\right)$

The summarizing function presented in equation 12 includes the iGPS model, which converts $\mathbf{e}_{i}$ and $\mathbf{r}_{T_{i}}$ to $\mathbf{t}_{i}$ and $\mathbf{r}_{i}$, as explained earlier.

\section{Validation: comparison of virtual and real measurements}

According to GUM [21], only valid measurement process models can be used for measurement uncertainty determination. For this reason, this section deals with design of experiments and the validation of the developed virtual iGPS. The goal of the experiments is to cover all impact factors to the iGPS measurement uncertainty with their parameter space, which were identified in Sect. 3.1.

\subsection{Experimental design}

There are three experiments that focus on individual subgoals following a so-called design of experiment (DoE) [28]: (i) exploring the configuration parameter space to validate that the virtual iGPS can be applied to arbitrary configurations, (ii) identifying standard uncertainties of the model's input variables and the effects through calibration, and (iii) obtaining additional validation data through receiver displacement. An overview of the experimental design is depicted in Fig. 4.

There is theoretically an infinite number of options to configure the iGPS in the 3D space. For this reason, the first experiment considers the configuration by applying a 2-level full factorial experimental design where transmitters are placed in a circular layout at the same height around a receiver (type i5 Integrated Sensor) in the circle center. The obtained measurement data is used to perform a validation by comparing real and modeled measurement values. The number of transmitters is set to three or six. The transmitter layout is equally distributed $(\mathrm{O})$ or a $\mathrm{C}$-shape $(\mathrm{C} /$ semicircular) which corresponds to most layouts in industrial assembly. The elevation angle between the transmitter plane and the receiver is 0 ŕ or 30 r. The distance between the transmitters and the receiver is set to 3 or $7 \mathrm{~m}$. The levels of the factors are determined based on technical limitations of the iGPS and knowledge gained from previous work of the authors. For each combination of factor levels, the system 

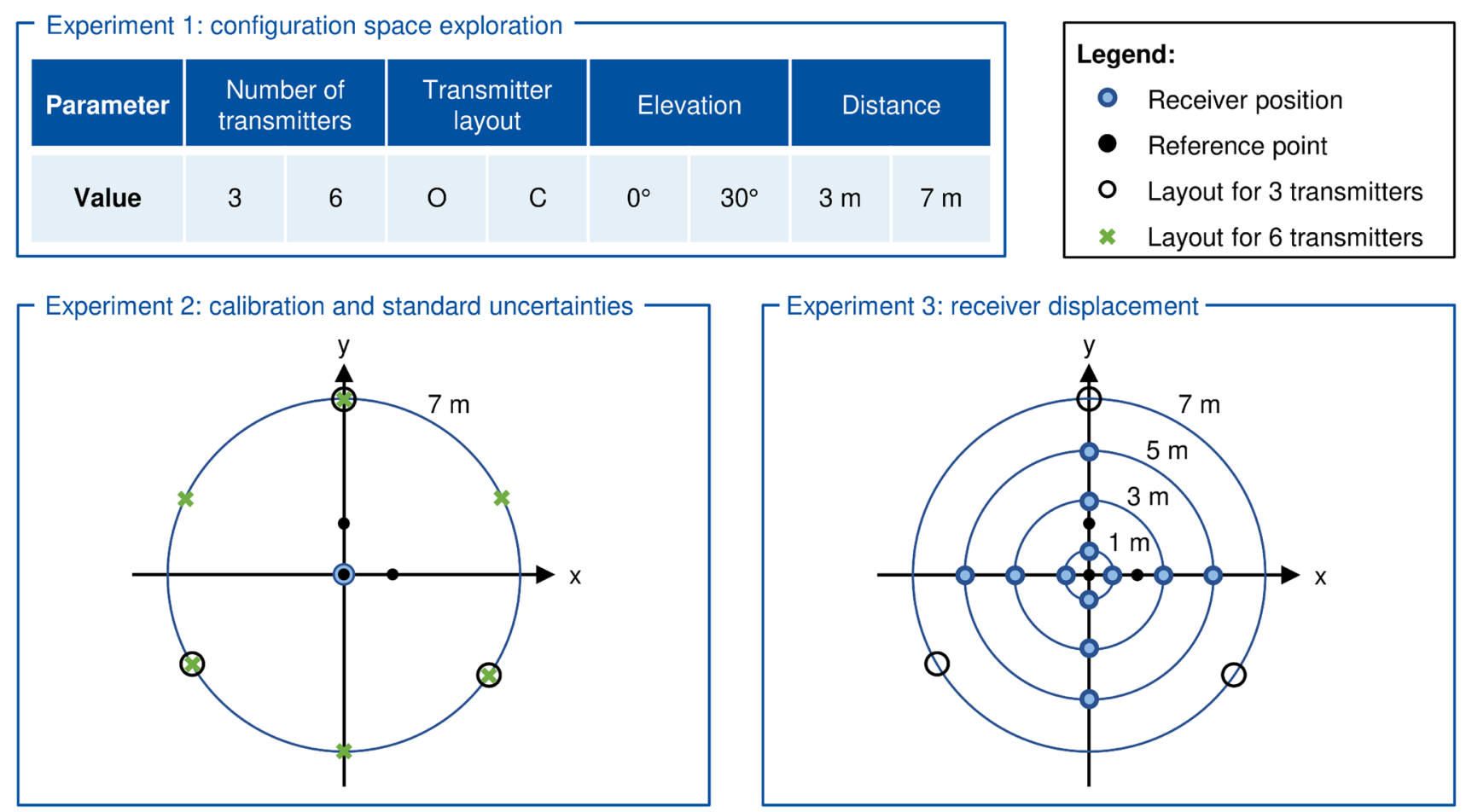

Fig. 4 iGPS experiments with variable configurations to obtain validation data and to determine standard uncertainties of impact factors

is calibrated manually using an i6 Long Reach Probe (handheld device) and three reference points ( 0.5 inch nests) to define a world coordinate system. 400 samples (repetition of single measurements) are performed to allow statistical analysis. The number of samples corresponds to the recommended number according to DoE for a standard deviation of $0.1 \mathrm{~mm}$ and relevant measurement value increments of $0.01 \mathrm{~mm}$.

In the second experiment, the standard uncertainties of the model's input variables are determined as required by GUM [21]. To derive standard uncertainties, repetitive measurements are conducted for each input variable. A single measurement comprises the Euler poses of all transmitters, determined after a calibration routine. The results of the experiment are crucial to the development of the measurement uncertainty model in Sect. 5.1. The transmitter layout is similar to the first experiment. The transmitters are placed in a circular layout at the same height with a distance of $7 \mathrm{~m}$ from the receiver, which is placed at the circle center with an elevation angle of 0 r. Since the impact of varying transmitter numbers in calibration is unknown, the standard uncertainties for three and six transmitters are investigated with 20 repetitions for each layout. To analyze the uncertainty of the transmitter positions and orientations determined by the calibration, the three reference points from experiment 1 are used to define a reference coordinate system with an i6 Long Reach Probe. Apart from the transmitter pose, the standard uncertainty of the LOS is determined by using the same configurations with 400 samples for each transmitter. By considering three and six transmitters for the different configurations, it can be discarded that the standard uncertainty of LOS is based on the specific behavior of a single transmitter and the number of transmitters.

In experiment one and two, the receiver is always placed in the center of the circle. To allow validation with unequal distances between transmitters and receivers, as usually found in industrial assembly, a third experiment is performed where the receiver is displaced. Starting from a distance of $1 \mathrm{~m}$ from the center of the circle, the receiver is displaced in increments of $2 \mathrm{~m}$ (from $1 \mathrm{~m}$ to $5 \mathrm{~m}$ ) in four directions (positive/negative $x$-direction and positive/negative $y$-direction). These receiver positions are obtained for two different heights: the transmitter plane (equals a height of $0 \mathrm{~m}$ ) and $2 \mathrm{~m}$ above the transmitter plane (in positive z-direction). Similar to the first experiment, all experiments are performed 400 times using three transmitters with a circular layout and a radius of $7 \mathrm{~m}$. Figure 5 shows a setup of the configuration experiments using 6 transmitters in a C-shape layout with an elevation angle of 30r and a receiver-transmitter distance of $7 \mathrm{~m}$. 
Fig. 5 Experimental environment showing an iGPS configuration with six transmitters, $\mathrm{C}$-shape layout, an elevation angle of 30 r̆ and a receivertransmitter distance of $7 \mathrm{~m}$

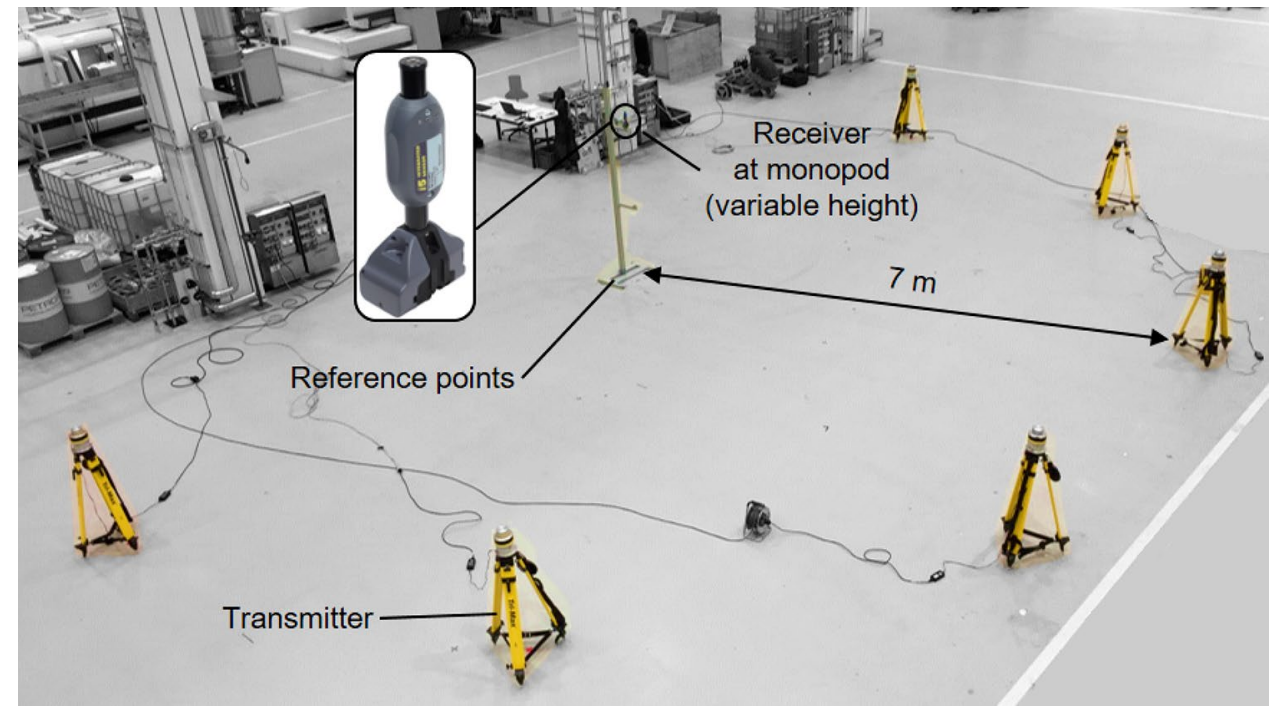

\subsection{Experimental results}

In the following, the data of experiment 1 and 3 are used to validate the developed iGPS measurement model. The data from experiment 2 are used in Sect. 5.2 to determine the standard uncertainties of the model's input variables. To validate the virtual iGPS, the following requirements for the measurement model will be verified:

- Mean value equality to the measured values of the measurement system

- Equality of variance to the measured values of the measurement system

Since the findings from literature $[15,17,20,34]$ confirm a correlation between iGPS measurement uncertainty and configuration, Welch's $t$ test [47] is used to validate the mean value equality. This test is suitable for mean value comparisons, where a variance equality cannot be assumed [35]. It is shown that Welch's $t$ test offers similar results to that of a standard t-test for homogeneous variances while keeping its robustness for unequal variances [22]. Therefore, Welch's t-test is preferred over the standard t-test in this work. Levene's test [25] is applied to validate the equality of variances. The validation is performed for the 2-norm of the measurement points (receiver positions). Both tests are performed for null hypotheses that the model and the measurement data do not have significant differences in mean and variance. For $p$-values greater 0.05 the respective null hypotheses can be retained. Figure 6 shows the results of the statistical tests for data of experiment 1 with a level of significance of $\alpha=0.05$. It can be seen that the measurement model achieves equality of means and variances for 13 of 16 configurations. The validation of the requirements for the measurement model can additionally be performed for the data from experiment 3. The results are shown in Fig. 7. For these data, the measurement model meets the requirements without exception. The results of the tests emphasize that the measurement model makes valid predictions with a significance level $\alpha=0.05$ for $\sim 81 \%$ of the experiment 1 data and for $\sim 100 \%$ of the experiment 3 data. According to GUM [21], the degree of accuracy of the measurement model should be in the range of the accuracy of the measurement result. This is given for the developed model of the measurement as the validation results underline and thus, the measurement model is suitable for the measurement uncertainty determination. Wasserstein et al. summarize that the validation of a model based on the p-value alone is not recommended [46]. Therefore, Sect. 5.2 examines the plausibility of the measurement uncertainty model results against existing knowledge from literature as proposed by Sargent [37].

\section{Results: measurement uncertainty determination for variable configurations}

In the following, a measurement uncertainty model is derived from the measurement model developed in Sect. 3.2. The standard uncertainties $u\left(x_{j}\right)$ of the input variables $x_{j}$ are initially determined, followed by postulating the measurement uncertainty model.

\subsection{Measurement uncertainty model}

For the LOS (with regard to the transmitter coordinate system) $\mathbf{r}_{T}$, the standard uncertainties can be subdivided into the spatial directions with:

$u\left(\mathbf{r}_{T}\right)=\left[\begin{array}{lll}u\left(r_{T, x}\right) & u\left(r_{T, y}\right) & u\left(r_{T, z}\right)\end{array}\right]^{T}$ 


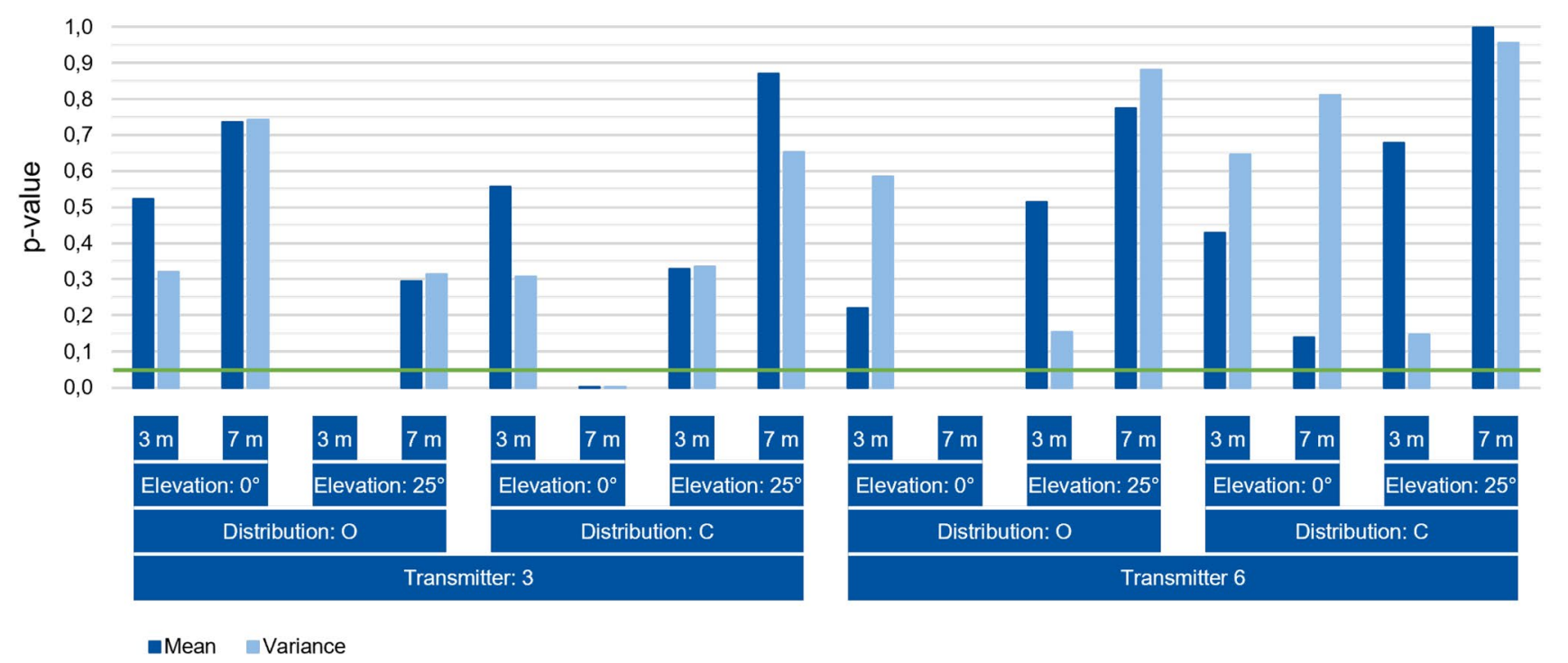

Fig. 6 Measurement model validation with experiment 1 data-level of significance $\alpha=0.05$ marked green

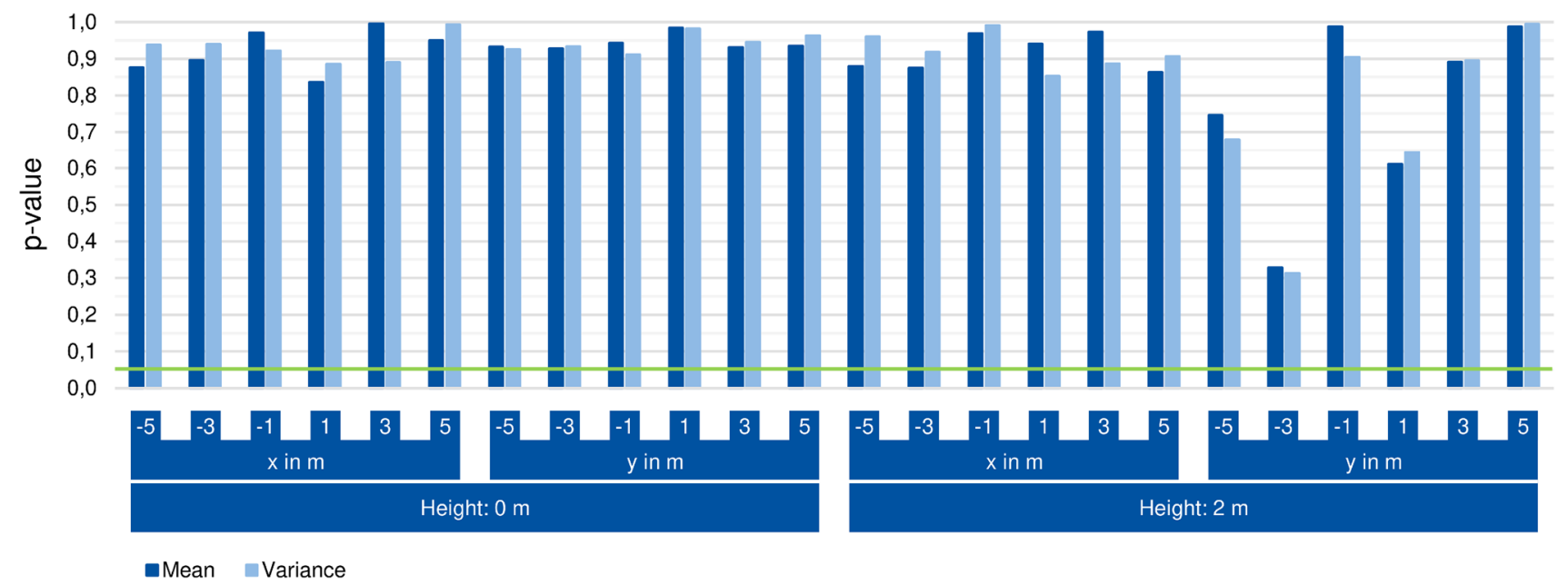

Fig. 7 Measurement model validation with experiment 3 data-level of significance $\alpha=0.05$ marked green

The standard uncertainties of the LOS can be determined based on the data from experiment 1 presented in Sect. 4.1. For this purpose, the standard uncertainties of the LOS can be calculated for each of the 16 configurations. The mean over all configuration experiments delivers the standard uncertainties of the LOS:

$u\left(\mathbf{r}_{T}\right)=\left[\begin{array}{lll}0.0072 \mathrm{~mm} & 0.0062 \mathrm{~mm} & 0.0052 \mathrm{~mm}\end{array}\right]^{T}$

The standard uncertainty of the Euler pose $\mathbf{e}$ is represented by its components:

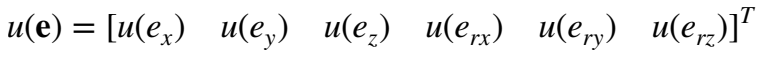

Using the data of experiment 2, the standard uncertainties of the Euler pose can be calculated depending on the number of transmitters. Table 2 gives an overview of the standard uncertainties for three and six transmitters derived from ten repetitions. The results show that the standard uncertainties decrease with increasing number of transmitters. The standard uncertainties of translation are reduced by an average of $72.3 \%$ by increasing the number from three to six transmitters. The standard uncertainties of rotation decrease by an average of $64.4 \%$.

Quinders [34] performed experiments to determine the standard uncertainty of the Euler pose for a fixed number of six transmitters. In this work, the standard uncertainty is also calculated for three transmitters following the design of experiment two. By conservatively assuming a linear relationship, a model is derived by interpolation for data points with three and six transmitters. Following this idea, the effect on the 
Table 2 Standard uncertainty of the transmitter Euler pose derived from data of experiment

\begin{tabular}{lllllll}
\hline $\begin{array}{l}\text { Trans- } \\
\text { mitters }\end{array}$ & $\begin{array}{l}\mathrm{u}\left(\mathrm{e}_{\mathrm{x}}\right) \\
\text { in mm }\end{array}$ & $\begin{array}{l}\mathrm{u}\left(\mathrm{e}_{\mathrm{y}}\right) \\
\text { in mm }\end{array}$ & $\begin{array}{l}\mathrm{u}\left(\mathrm{e}_{\mathrm{z}}\right) \\
\text { in mm }\end{array}$ & $\begin{array}{l}\mathrm{u}\left(\mathrm{e}_{\mathrm{ex}}\right) \\
\text { in r }\end{array}$ & $\begin{array}{l}\mathrm{u}\left(\mathrm{e}_{\mathrm{ey}}\right) \\
\text { in } \check{\mathrm{r}}\end{array}$ & $\begin{array}{l}\mathrm{u}\left(\mathrm{e}_{\mathrm{ez}}\right) \\
\text { in } \check{\mathrm{r}}\end{array}$ \\
\hline 3 & 1.1705 & 1.1197 & 0.7252 & 0.02868 & 0.01354 & 0.01349 \\
6 & 0.1867 & 0.2157 & 0.3479 & 0.00385 & 0.00659 & 0.00605 \\
\hline
\end{tabular}

uncertainty through calibration can be determined for numbers of transmitters between three and six $n_{T} \in[3 ; 6]$ :

$$
\begin{aligned}
& u\left(e_{x}\right)=-0.3279 \mathrm{~mm} \cdot n_{T}+2.1543 \mathrm{~mm} \\
& u\left(e_{y}\right)=-0.3013 \mathrm{~mm} \cdot n_{T}+2.0236 \mathrm{~mm} \\
& u\left(e_{z}\right)=-0.1257 \mathrm{~mm} \cdot n_{T}+1.1025 \mathrm{~mm} \\
& u\left(e_{e x}\right)=-0.0082758^{\circ} \cdot n_{T}+0.053505^{\circ} \\
& u\left(e_{e y}\right)=-0.0023208^{\circ} \cdot n_{T}+0.020510^{\circ} \\
& u\left(e_{e z}\right)=-0.0023622^{\circ} \cdot n_{T}+0.020578^{\circ}
\end{aligned}
$$

Since a minimum number of three LOS are required for a valid measurement, fewer transmitters than three are not considered. Therefore, the linear model for the standard uncertainties of the Euler pose has a validity for a number of transmitters between three and six. If the standard uncertainty would be determined for a configuration calibrated with 10 transmitters, $n_{T}$ is set to $n_{T}=6$, since the model only has a validity up to a number of 6 transmitters.

According to GUM [21] the combined squared standard uncertainty $u_{c}(y)^{2}$ of a numerical measurement model with $j$ input values may be calculated by:

$$
\begin{gathered}
u_{c}(y)^{2}=\sum_{j=1}^{N}\left[\frac{1}{2} \cdot f\left(x_{1}, \ldots, x_{j}+u\left(x_{j}\right), \ldots, x_{N}\right)\right. \\
\left.-\frac{1}{2} \cdot f\left(x_{1}, \ldots, x_{j}-u\left(x_{j}\right), \ldots, x_{N}\right)\right]^{2}
\end{gathered}
$$

Mathematically, equation 22 presents a numerical differentiation of the measurement model in respect to each input value. For $i$ transmitters the measurement model developed in Sect. 3.2 may be formulated as:

$\mathbf{p}_{\min }=f\left(r_{T, x_{i}}, r_{T, y_{i}}, r_{T, z_{i}}, e_{x_{i}}, e_{y_{i}}, e_{z_{i}}, e_{e x_{i}}, e_{e y_{i}}, e_{e z_{i}}\right)$

For $\mathrm{j}$ input values, the combined squared standard uncertainty of $\mathbf{p}_{\min }$ may then be denoted as:

$$
\begin{aligned}
u_{c}\left(\mathbf{p}_{\text {min }}\right)^{2}= & \sum_{j=1}^{N}\left[\frac{1}{2} \cdot f\left(r_{T, x_{j}}, \ldots, e_{x_{j}}+u\left(e_{x}\right), \ldots, e_{e z_{j}}\right)\right. \\
& \left.-\frac{1}{2} \cdot f\left(r_{T, x_{j}}, \ldots, e_{x_{j}}-u\left(e_{x}\right), \ldots, e_{e z_{j}}\right)\right]^{2}
\end{aligned}
$$

Equation 24 presents the complete measurement uncertainty model. In order to illustrate the model, an example of the model equation for three transmitters is given:

$$
\begin{aligned}
\mathbf{p}_{\min }= & f\left(r_{T, x_{1}}, r_{T, y_{1}}, r_{T, z_{1}}, e_{x_{1}}, e_{y_{1}}, e_{z_{1}}, e_{r x_{1}}, e_{e y_{1}}, e_{e z_{1}},\right. \\
& r_{T, x_{2}}, r_{T, y_{2}}, r_{T, z_{2}}, e_{x_{2}}, e_{y_{2}}, e_{z_{2}}, e_{r x_{2}}, e_{e y_{2}}, e_{e z_{2}}, \\
& \left.r_{T, x_{3}}, r_{T, y_{3}}, r_{T, z_{3}}, e_{x_{3}}, e_{y_{3}}, e_{z_{3}}, e_{r x_{3}}, e_{e y_{3}}, e_{e z_{3}}\right)
\end{aligned}
$$

For three transmitters, the combined squared standard uncertainty can then be calculated with a numerical differentiation of equation 25 in respect to its input values:

$$
\begin{aligned}
u_{c}\left(\mathbf{p}_{\text {min }}\right)^{2}= & {\left[\frac{1}{2} \cdot f\left(r_{T, x_{1}}+u\left(r_{T, x}\right), \ldots, e_{e z_{3}}\right)\right.} \\
& \left.-\frac{1}{2} \cdot f\left(r_{T, x_{1}}-u\left(r_{T, x}\right), \ldots, e_{e z_{3}}\right)\right]^{2} \\
& +\ldots \\
& +\left[\frac{1}{2} \cdot f\left(r_{T, x_{1}}, \ldots, e_{e z_{3}}+u\left(e_{e z}\right)\right)\right. \\
& \left.-\frac{1}{2} \cdot f\left(r_{T, x_{1}}, \ldots, e_{e z_{3}}-u\left(e_{e z}\right)\right)\right]^{2}
\end{aligned}
$$

The measurement uncertainty model developed in this section is implemented in Matlab. Since the measurement uncertainty model is based on a least-square algorithm (see Sect. 3.2), it is reasonable to consider the computation time. Investigations for a setup with three transmitters and one receiver demonstrate a computation time of $0.0071 \mathrm{~s}$ with an AMD FX-8350 processor using single core calculation. For a setup with six transmitters and one receiver, the computation time increases to a value of $0.0225 \mathrm{~s}$.

\subsection{Comparison between model output and literature knowledge}

Since the measurement uncertainty model developed follows the GUM methodology [21] and is therefore based on a positively validated measurement model, the measurement uncertainty model is implicitly valid. Due to the fact that only the p-value for mean and variance equality were considered for the validation of the measurement model, 
the plausibility of the measurement uncertainty model is assessed in more detail by comparing existing knowledge from the literature and the model results, as suggested by Sargent [37]. Therefore, model results are represented by heatmaps showing the spatial distribution of measurement uncertainty within the investigated parameter ranges (see Fig. 8-10).

To generate these heatmaps, specific configurations are manually defined first. A maximum working range (x-axis, $\mathrm{y}$-axis) is defined, for which an equally distributed grid is subsequently generated. Each grid point serves as a receiver position for which the measurement uncertainty is calculated. Using the user given transmitter-poses $\mathbf{e}_{i}$, the transmitter-specific LOS $\mathbf{r}_{T_{i}}$ are calculated for each transmitter and grid point, forming the input values for the measurement uncertainty determination. The uncertainties are displayed in heatmaps as a function of the $\mathrm{x}$ - and $\mathrm{y}$-coordinates. In this work, the $2 \mathrm{D}$ representation of $u_{c}$ refers to the height plane (z-coordinate) of the transmitters, but could also be adjusted arbitrarily.

Schmitt et al. [38] and Quinders [34] have shown that a more uniform spatial distribution of transmitters around a receiver results in a lower measurement uncertainty. The two heatmaps in Fig. 8 compare configurations with a transmitter distribution over 360r and 180 r as examples of uniform and non-uniform distributions. The line for $\mathrm{u}_{\mathrm{c}}=0.72 \mathrm{~mm}$ illustrates that the absolute measurement range that fulfills $\mathrm{u}_{\mathrm{c}}<0.72 \mathrm{~mm}$ increases applying a distribution over 360 r.

According to works from [26, 34, 43], measurements performed closer to the transmitters or with a smaller number of lines of sight have a higher measurement uncertainty. Starting from a minimum of measurement uncertainty in the center of the area surrounded by the transmitters, the measurement uncertainty increases in the direction of the transmitters, as illustrated by the two heatmaps in Fig. 9 . The comparison of the configurations emphasizes that if the number of lines of sight decreases from six (left heatmap) to three (right heatmap), the minimum measurement uncertainty increases from $0.54 \mathrm{~mm}$ to $0.76 \mathrm{~mm}$.

According to Landeta et al. [23], the calibration has the largest contribution to the overall uncertainty budget (83\%). The left heatmap in Fig. 10 shows $u_{c}$ considering three lines of sight and a calibration with three transmitters. The right heatmap shows $\mathrm{u}_{\mathrm{c}}$ considering three lines of sight and a calibration with six transmitters. By increasing the number of transmitters from three to six, the minimum measurement uncertainty decreases by $82 \%$ from 4.2 to $0.7 \mathrm{~mm}$, which underlines the strong influence of the calibration.

Summarizing the results of the comparisons, the model behavior matches the expected behavior according to the knowledge from literature considering the investigated parameter range. For this reason, the results of the measurement uncertainty model are interpreted as plausible, which confirms the model validity.

\subsection{Application to airplane assembly and investigation of process capability}

The validated measurement uncertainty model is applied to wing assembly, which is part of the final airplane assembly. The application of the measurement uncertainty model illustrates how measurement processes can be improved in terms of process capability to verify narrow tolerances in the millimeter range at large distances. Figure 11 shows the simulated wing assembly process on the left, where the joining partners (wing and fuselage parts) are placed on automated guided vehicles (AGV). The simulation is based on the software Matlab (Simulink 3D Animation [3]) which
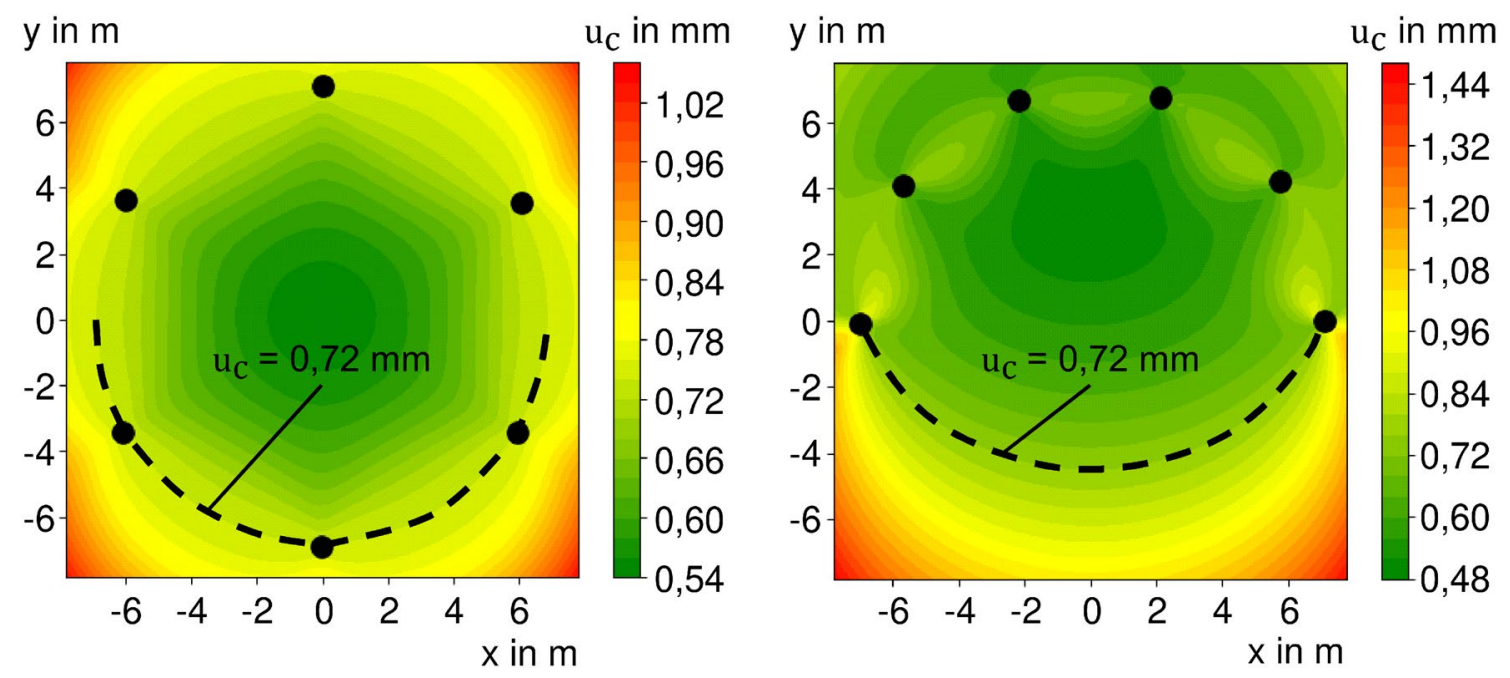

Fig. 8 Effect of uniform and non-uniform transmitter distributions on measurement uncertainty 

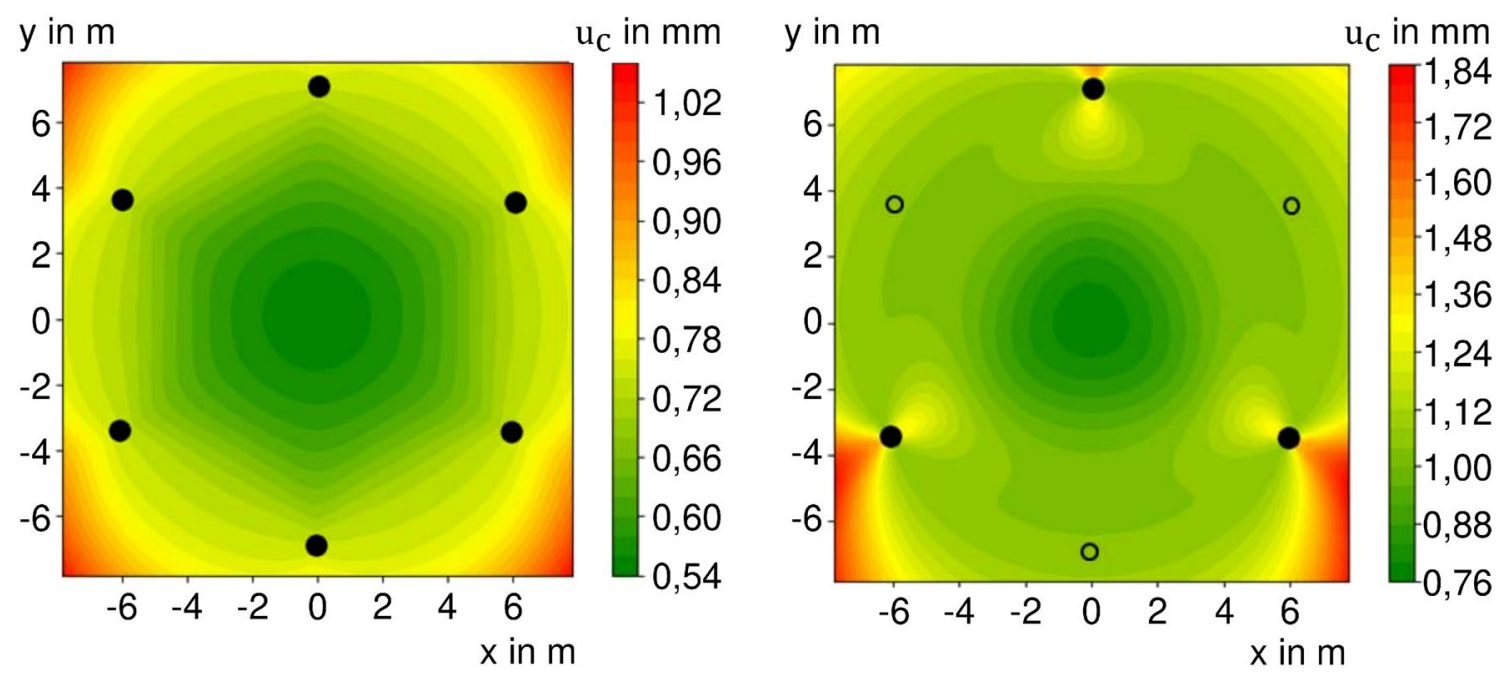

Fig. 9 Effect of decreasing number of lines of sight on measurement uncertainty
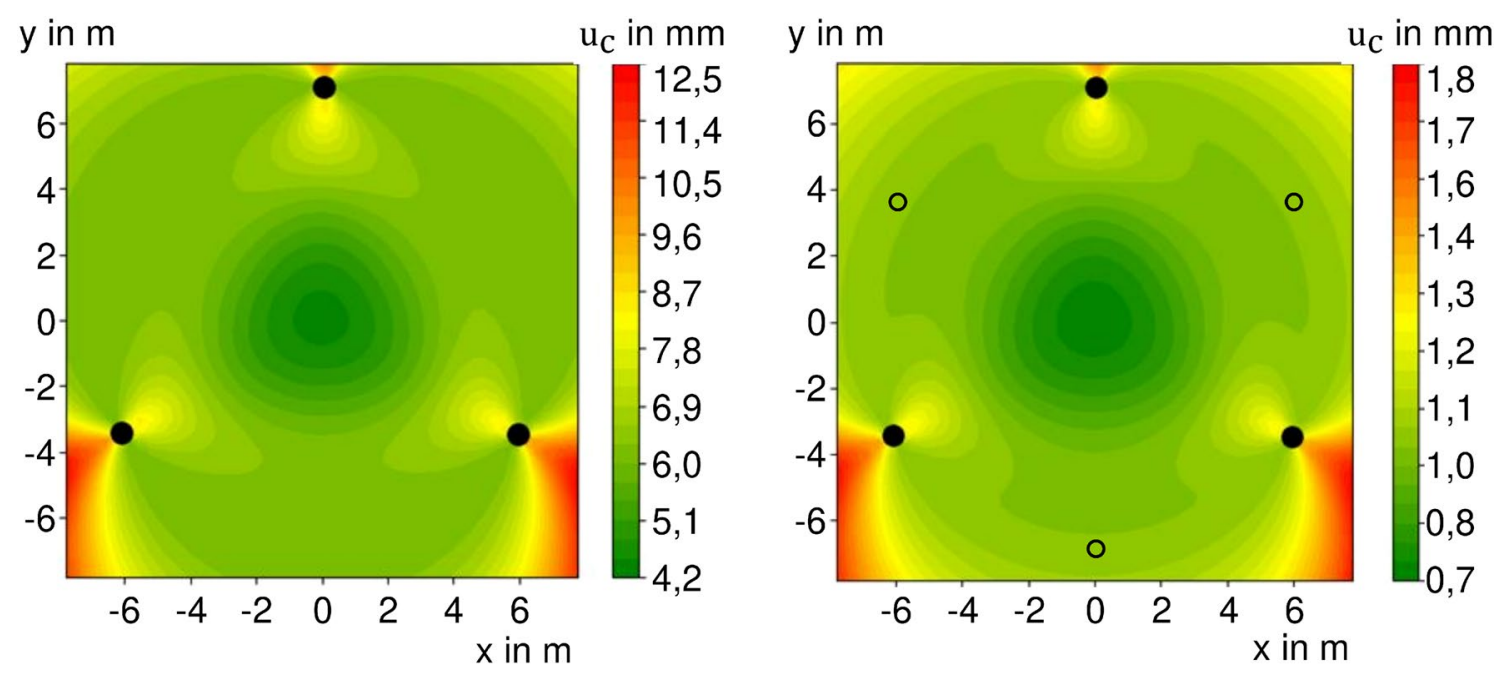

Fig. 10 Effect of different numbers of transmitters in calibration

also enables simulations of dynamic processes and collision detection of the LOS. The assembly layout mimics the state of the art of airplane manufacturers currently attempting to implement line-less, mobile assembly paradigms [1, 6, 31, 44]. The right picture in Fig. 11 shows a real wing assembly where the iGPS has been deployed [2].

To verify that the measurement model can be used to decrease uncertainties for capable measurement processes, two different iGPS configurations are compared: The first configuration represents a worst-case configuration using three transmitters whereas the second configuration comprises six transmitters, which is the upper limit due to the validated parameter range of the measurement model. To enable application to the spatial dimensions of airplane assembly, uncertainty values are extrapolated regarding the impact factors distance and transmitter distribution, which is not allowed if model validity is assumed according to GUM [21]. Therefore, the use case relies on a partially non-validated model, but emphasizes the potential of virtual measurements and correlating uncertainty determinations.

There are seven static receivers to be measured within the assembly process, four on the fuselage and three on the wing (see Fig. 11 on the left). For both configurations, the transmitters are distributed manually according to the technical limitations of the iGPS (see Sect. 2.2) at a height of $5 \mathrm{~m}$. In terms of a robust calibration, it is assumed that the transmitters may not be placed on any part of the airplane or on mobile equipment (e.g. AGVs), but may be placed on stationary work platforms. 
After the manual transmitter positioning, the developed measurement uncertainty model is used to create $2 \mathrm{D}$ heatmaps (at the receiver height of $5.5 \mathrm{~m}$ ) to illustrate the measurement uncertainty distribution over the assembly station. The heatmap of the configuration with three transmitters is depicted in Fig. 12. Obviously, there is an inhomogeneous measurement uncertainty distribution over the assembly station area, with a minimum between the transmitters of about $6.65 \mathrm{~mm}$ and increasing values towards the boundaries of the assembly station up to $50.95 \mathrm{~mm}$. Figure 13 shows the

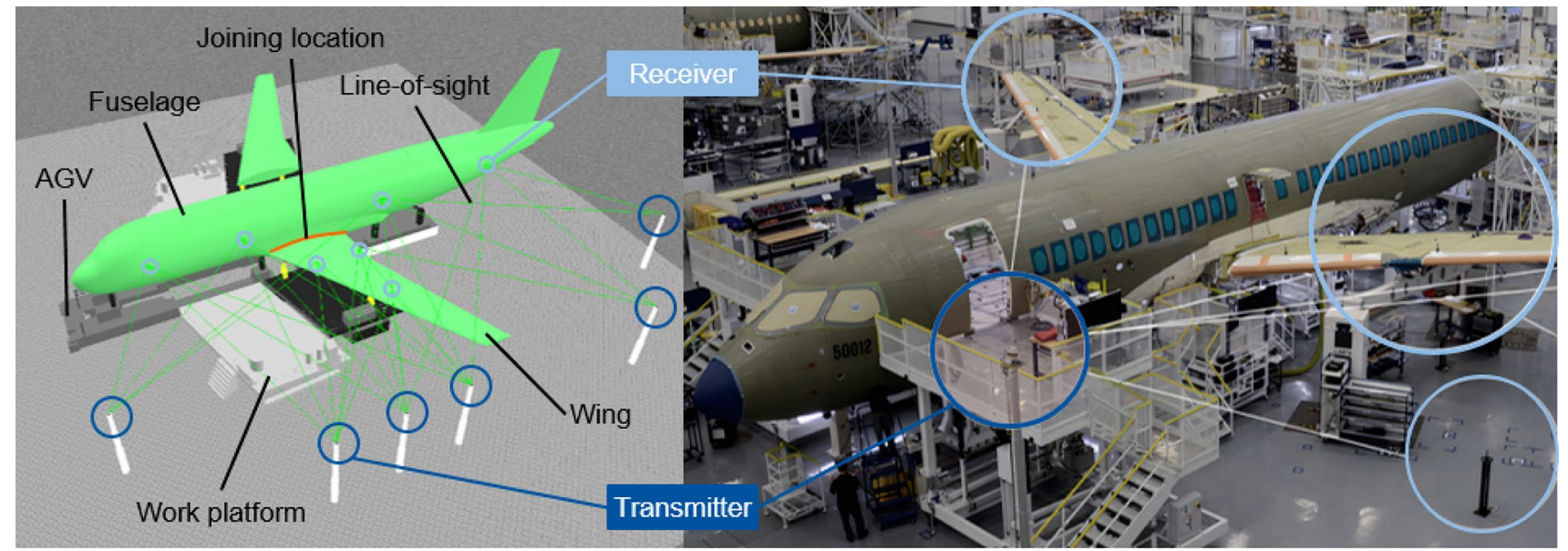

Fig. 11 Simulated (left figure) and real (right figure [2]) wing assembly of airplanes using iGPS to join wing and fuselage within required assembly tolerances

Fig. 12 2D distribution of the iGPS measurement uncertainty at the receiver level (height of $5 \mathrm{~m}$ ) using three transmitters
Fig. 13 2D distribution of the iGPS measurement uncertainty at the receiver level (height of $5 \mathrm{~m}$ ) using six transmitters
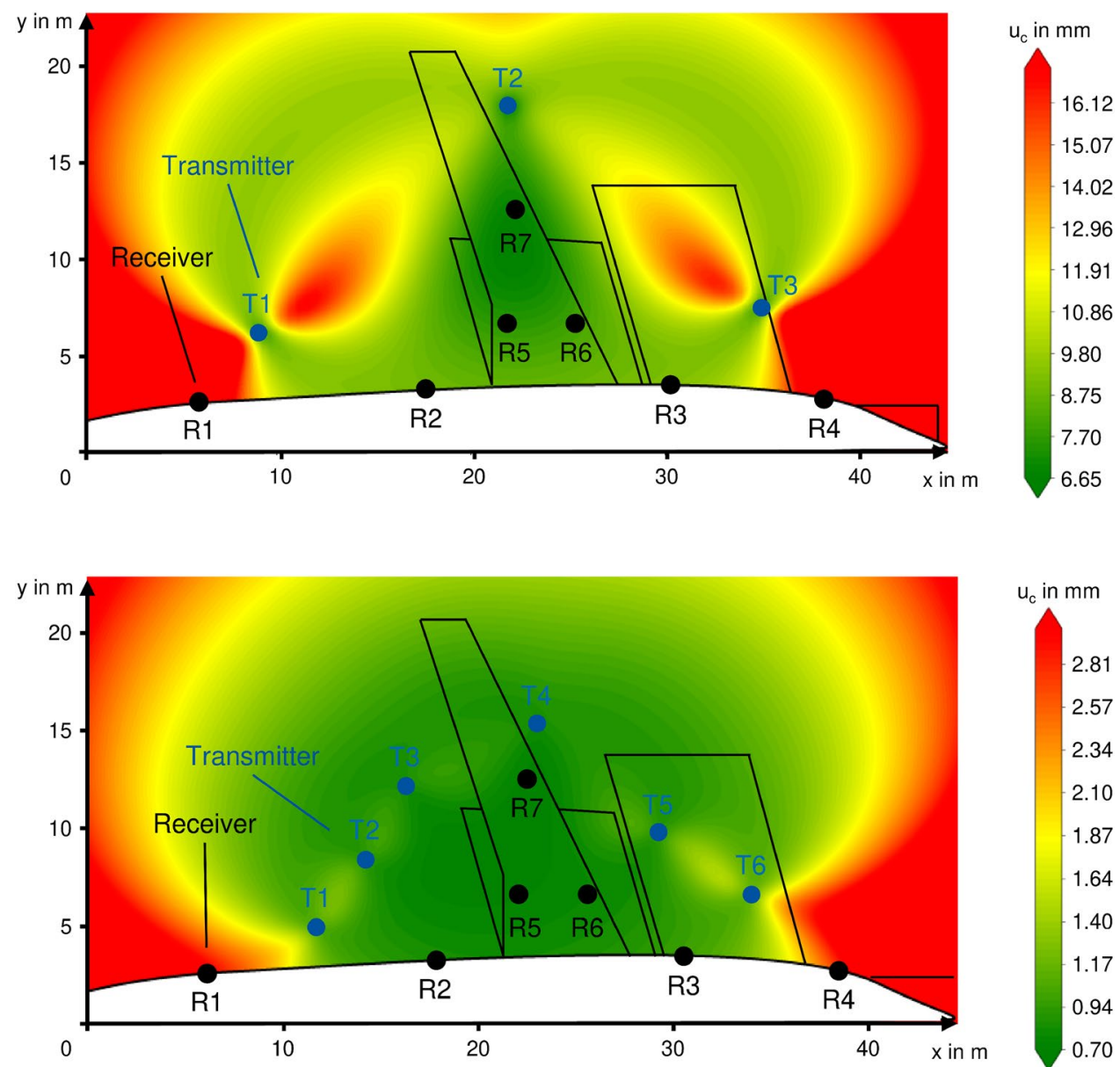
configuration with six transmitters, in which the measurement uncertainty follows the same tendency regarding the minimum and the values at the boundaries of the assembly station. It can be seen that areas of high measurement uncertainty (see isolated red areas next to transmitter one and three in Fig. 12) can be eliminated by using six transmitters. In comparison to the three transmitter configuration, the absolute values of the measurement uncertainty decrease to a minimum of about $0.70 \mathrm{~mm}$ and a maximum of about $6.15 \mathrm{~mm}$. (The program, including the source code of the virtual indoor-GPS and the measurement uncertainty model to generate 2D heatmaps, can be downloaded from [32].)

An overview of all seven measurement uncertainties at the receivers for both configurations is given in Fig. 14. On average, there is a difference of $11.07 \mathrm{~mm}$ between the threetransmitter configuration $(12.48 \mathrm{~mm})$ and the six-transmitter configuration $(1.41 \mathrm{~mm})$. Based on the formulation of the uncertainty contributions in Eq. 25, the root cause for the difference can be seen: $99 \%$ of the difference results from the uncertainty of the calibration, whereas only $1 \%$ is due to the uncertainty of the configuration. Apart from the knowledge about the absolute values, the uncertainties obtained from virtual iGPS measurements enable an a priori determination whether the measurement process is capable or not. According to Dietrich [10], a measurement process is not capable if the ratio between measurement uncertainty and tolerance is greater than 0.3. Figure 14 shows the ranges for capable (green highlighted) and non-capable (red highlighted) measurements, assuming a tolerance of $3 \mathrm{~mm}$. Considering the given six-transmitter configuration, there are four out of seven receivers in the capable range and can be used for assembly process.

\section{Conclusion and outlook}

In this work, a virtual indoor-GPS (iGPS) was proposed, allowing a priori determination of the measurement process capability to assist operators in assembly. It was found out that the iGPS as a distributed large-scale metrology system fits to current trends in large-scale product assembly, providing highly accurate positioning data in reconfigurable environments such as Line-less Mobile Assembly Systems. Since the measurement uncertainty of the iGPS depends on the spatial distribution of the transmitters, the virtual iGPS was developed to mimic real measurements and to derive the measurement uncertainties according to GUM. After a successful validation, the virtual iGPS was applied to an airplane wing assembly with seven receivers and a maximum number of six transmitters. The use case showed that measurement uncertainties can be decreased from 12.48 to $1.41 \mathrm{~mm}$ on average by adding transmitters and varying transmitter positions. Considering given assembly tolerances, measurement process capabilities for iGPS receivers can be determined a priori. It was pointed out that operators who manually position iGPS transmitters face the challenge
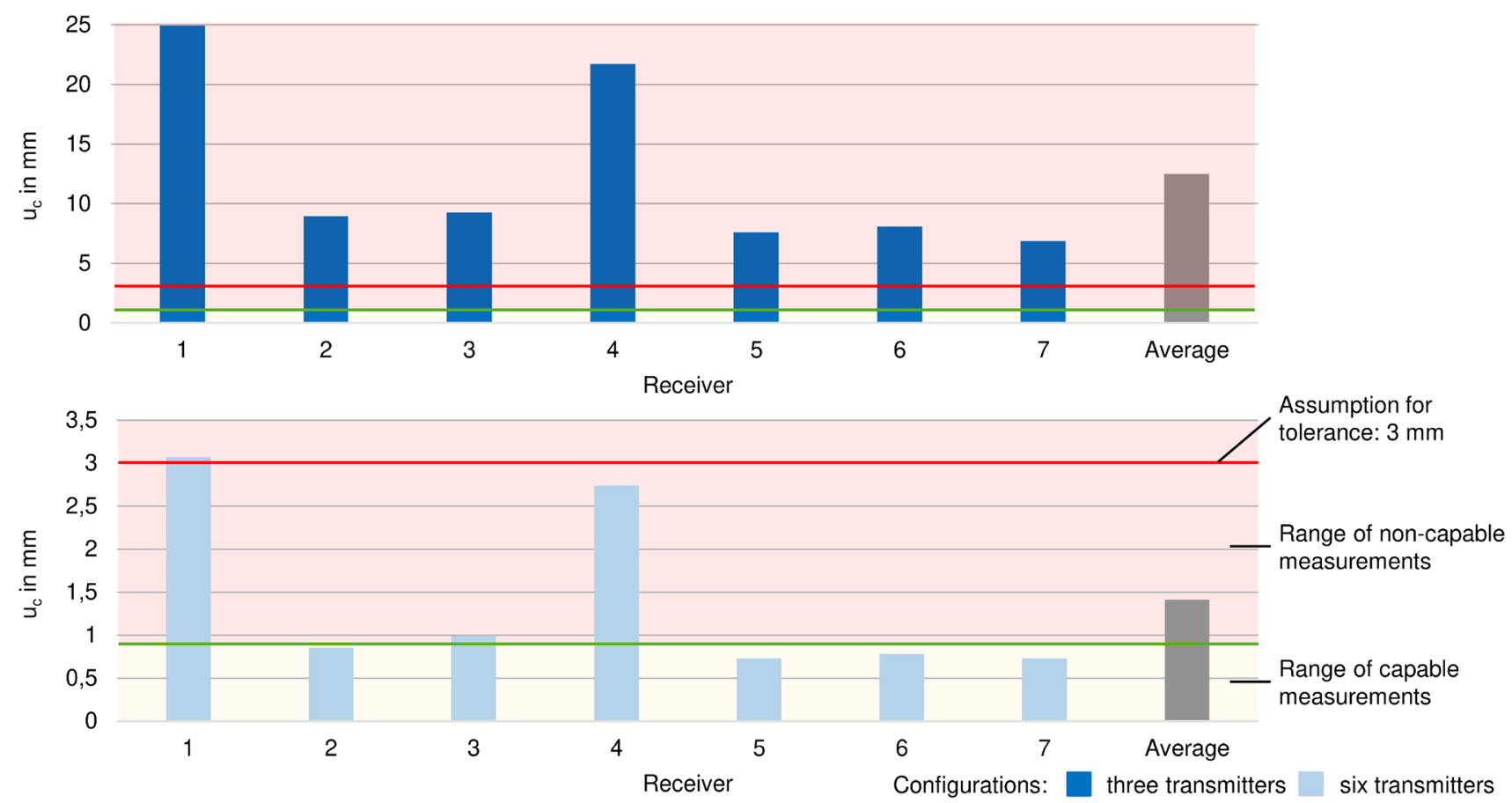

Fig. 14 Comparison of resulting measurement uncertainties of the receivers using three and six transmitters 
of an inhomogeneous uncertainty distribution, emphasizing the need for a virtual iGPS as a planning tool.

To address this challenge, the authors will focus on automated transmitter positioning for distributed large-scale metrology systems in future works. Thereby, global optimization algorithms can consider the developed measurement uncertainty model as an objective function. Thereby, transmitter positions can be automatically determined within the assembly station that cause minimal measurement uncertainties at the receivers. Based on this result, the operator can decide in a time-efficient manner whether to add or remove transmitters enabling demand-oriented and capable configurations. Since the calibration has the greatest effect on measurement uncertainty, other calibration techniques such as monument-based calibration will be investigated. Also, the effect of systematic measurement errors on the measurement uncertainty will be investigated using a laser tracker as a reference system. In addition, the proposed analytical approach forms the basis for modeling other optical and distributed measurement systems, such as laser tracker networks and laser tracers.

Acknowledgements This work is part of the research project "iVeSPA" that is funded by the German Federal Ministry for Economic Affairs and Energy (BMWi) within the "LuFo - the Federal Aviation Research Programme" (funding number: 20X1708G) and supported by the project management agency German Aerospace Center (DLR-PT). The author is responsible for the content of this publication.

Funding Open Access funding enabled and organized by Projekt DEAL.

\section{Declarations}

Conflict of interest The authors declare that they have no conflict of interest.

Open Access This article is licensed under a Creative Commons Attribution 4.0 International License, which permits use, sharing, adaptation, distribution and reproduction in any medium or format, as long as you give appropriate credit to the original author(s) and the source, provide a link to the Creative Commons licence, and indicate if changes were made. The images or other third party material in this article are included in the article's Creative Commons licence, unless indicated otherwise in a credit line to the material. If material is not included in the article's Creative Commons licence and your intended use is not permitted by statutory regulation or exceeds the permitted use, you will need to obtain permission directly from the copyright holder. To view a copy of this licence, visit http://creativecommons.org/licenses/by/4.0/.

\section{References}

1. A futuristic view of the 777 fuselage build (2014). http://www. boeing.com/features/2014/07/bca-777-fuselage-07-14-14.page

2. Industries - solutions for manufacturing and transportation (2021). https://7dkmetrology.com/
3. Simulink $3 \mathrm{~d}$ animation: Visualize dynamic system behavior in a virtual reality environment (2021). https://www.mathworks.com/ products/3d-animation.html

4. 7D Kinematic Metrology: Products - 6th generation technology further increases igps usability \& performance (2021). https:// 7dkmetrology.com/7dk-1/products

5. Brecher C, Schmitt RH, Behrens J, Ohlenforst M, Peterek M, Wennemer M (2016) Präzisionsfertigung von grossbauteilen. Maschinenmarkt 7(122):36-40

6. Briegleb, V.: Roboter im flugzeugbau: Airbus automatisiert a320-produktion (2019). https://www.heise.de/newsticker/meldu ng/Roboter-im-Flugzeugbau-Airbus-automatisiert-A320-Produ ktion-4544052.html

7. Chen Z, Du F (2017) Measuring principle and uncertainty analysis of a large volume measurement network based on the combination of igps and portable scanner. Measurement 104:263-277. https:// doi.org/10.1016/j.measurement.2017.03.037

8. Demeester F, Dresselhaus M, Essel I, Jatzkowski P (2011) Referenzsysteme für wandlungsfähige produktion. In: Brecher, C, Klocke F, Schmitt R, Schuh G (eds) Wettbewerbsfaktor Produktionstechnik: Aachener Perspektiven, pp 3-28

9. Depenthal C, Schwendemann J (2009) igps - a new system for static and kinematic measurements. In: Grün A, Kahmen H (eds) Optical 3-D measurement techniques VI, pp 131-140. Zürich

10. Dietrich E, Schulze A (2017) Eignungsnachweis von Prüfprozessen: Prüfmittelfähigkeit und Messunsicherheit im aktuellen Normenumfeld, 5. überarbeitete auflage edn. Hanser, München. https://doi.org/10.3139/9783446451711

11. Dobrev Y, Vossiek M, Christmann M, Bilous I, Gulden P (2017) Steady delivery: wireless local positioning systems for tracking and autonomous navigation of transport vehicles and mobile robots. IEEE Microwave Mag 18(6):26-37

12. Drouot A, Zhao R, Irving L, Sanderson D, Ratchev S (2018) Measurement assisted assembly for high accuracy aerospace manufacturing. IFAC-PapersOnLine 51(11):393-398

13. Forbes AB (2016) Reference model and algorithms for multistation coordinate metrology. In: 21st IMEKO World Congress Measurement in research and industry, pp. 1848-1853. Curran Associates, Red Hook

14. Franceschini F, Galetto M, Maisano D, Mastrogiacomo L, Pralio B (2011) Distributed large-scale dimensional metrology. New insights. https://doi.org/10.1007/978-0-85729-543-9

15. Guo S, Lin J, Ren Y, Yang L, Zhu J (2017) Study of network topology effect on measurement accuracy for a distributed rotarylaser measurement system. Opt Eng 56(9) (2017). https://doi.org/ 10.1117/1.OE.56.9.094101

16. Hedges T, Beliveau S, Pratt T, Slater R, Sobel J (2002) Aparatus and method for determining position: United states patent

17. Hughes B, Forbes A, Sun W, Maropoulos P, Muelaner J, Jamshidi J, Wang Z (2010) igps capability study

18. Hui Z, Xin P, Jipeng B, Linghui Y (2020) Research on the application of indoor gps in aircraft assembly. In: Zhu J (ed) 2019 International Conference on Optical Instruments and Technology: Optoelectronic Measurement Technology and Systems, Proceedings of SPIE, p. 49. SPIE, Bellingham, Washington, USA. https:// doi.org/10.1117/12.2543384

19. Hüttemann G, Buckhorst AF, Schmitt RH (2019) Modelling and assessing line-less mobile assembly systems. Procedia CIRP 81:724-729

20. Jingtao F, Xiang H, Qi Z, Ziyue Z, Zhufeng S (2021) Placement optimization of igps transmitter based on immune optimization algorithm. Laser Optoelectron Progress 58(12). https://doi.org/10. 3788/LOP202158.1112009 
21. Joint Committee for Guides in Metrology: Evaluation of measurement data - guide to the expression of uncertainty in measurement (01.09.2008). www.bipm.org

22. Kubinger KD, Rasch D, Moder K (2009) Zur legende der voraussetzungen des $\mathrm{t}$-tests für unabhängige stichproben. Psychol Rundsch 60(1):26-27. https://doi.org/10.1026/0033-3042.60.1.26

23. Landeta B, Suterio R, Trabasso GL (2011) A metrologycal analysis of the igps system focusing on its main sources of uncertainties. https://nanopdf.com/download/a-metrologycal-analysis-ofthe-igps-system-focusing-on-its-sources-of-uncertaint_pdf

24. Leach R, Bointon P, Feng X, Lawes S, Piano S, Senin N, SimsWaterhouse D, Stavroulakis P, Su R, Syam W, Thomas M (2019) Information-rich manufacturing metrology. In: Ratchev $\mathrm{S}$ (ed) Precision Assembly in the Digital Age, IFIP Advances in Information and Communication Technology. Springer, Cham, pp $145-157$

25. Levene H (1960) Robust tests for equality of variances. Contributions to probability and statistics: essays in honor of harold hotelling, pp 278-292

26. Maisano DA, Jamshidi J, Franceschini F, Maropoulos PG, Mastrogiacomo L, Mileham AR, Owen GW (2008) Indoor gps: system functionality and initial performance evaluation. Int J Manuf Res 3(3):335. https://doi.org/10.1504/IJMR.2008.019214

27. Montavon B, Peterek M, Schmitt R (2017) Communication architecture for multiple distributed large volume metrology systems. In: International symposium on systems engineering 2017

28. Montgomery DC (2019) Design and analysis of experiments, 10, ed. Wiley, Hoboken, NJ

29. Muelaner JE, Martin OC, Maropoulos PG (2013) Achieving low cost and high quality aero structure assembly through integrated digital metrology systems. Procedia CIRP 7:688-693

30. Mueller T, Huber M, Schmitt R (2020) Modelling complex measurement processes for measurement uncertainty determination. Int J Qual Reliab Manag 37(3):494-516. https://doi.org/10.1108/ IJQRM-07-2019-0232

31. Nicksch C, Kluge-Wilkes A, Huber M, Schmitt RH (2020) Global reference system for factory-wide integration of metrology enabling flexible automation in aeroplane assembly - requirements, concept and suitable technologies. Procedia Manuf 52:89-94

32. Nicksch C, Sabzehi M, Schmitt RH (2022) Virtual indoor-gps. GitLab. https://git-ce.rwth-aachen.de/christoph.nicksch/virtualindoor-gps.git

33. Pfeifer T (2015) Production Metrology. Oldenbourg Wissenschaftsverlag, Berlin, Boston. https://doi.org/10.1515/9783486810 424

34. Quinders S (2017) Virtueller prototyp zur optimierung und absicherung der konfiguration messtechnisch gestützter und roboterbasierter montagesysteme. Dissertation, RWTH Aachen University

35. Ruxton GD (2006) The unequal variance t-test is an underused alternative to student's t-test and the mann-whitney u test. Behav Ecol 17(4):688-690. https://doi.org/10.1093/beheco/ark016

36. Šafarevič IR, Remizov AO (2012) Linear algebra and geometry. Springer, Berlin. https://doi.org/10.1007/978-3-642-30994-6

37. Sargent, R.G.: Verification and validation of simulation models. Proceedings of the 2010 Winter Simulation Conference pp. 166$183(2010)$

38. Schmitt R, Nisch S, Schonberg A, Demeester F, Renders S (2010) Performance evaluation of igps for industrial applications. In: Mautz R (ed.) International Conference on Indoor Positioning and Indoor Navigation (IPIN), 2010, pp. 1-8. IEEE, Piscataway, NJ. https://doi.org/10.1109/IPIN.2010.5647630

39. Schmitt R, Wiederhold M, Damm J, Harding M, Jatzkowski P, Ottone R (2014) Cost-efficient measurement system analysis for small-batch production. Key Eng Mater 613:417-427. https://doi. org/10.4028/www.scientific.net/KEM.613.417

40. Schmitt, R.H., Eickhorst, D., Flöter, F.: Future assembly with distributed sensor services. In: T. Bergs, C. Brecher, R.H. Schmitt, Schuh G. (eds.) Turning Data Into Value, pp. 158-180 (2021). https://doi.org/10.24406/ipt-n-589615

41. Schmitt RH, Peterek M, Morse E, Knapp W, Galetto M (2016) Advances in large-scale metrology - review and future trends. CIRP Ann Manuf Technol 65:643-665. https://doi.org/10.1016/j. cirp.2016.05.002

42. Schmitt, R.H., Voigtmann, C.: Sensorinformationen als dienst - baustein der vernetzten produktion. tm - Technisches Messen 84(5) (2017). https://doi.org/10.1515/teme-2017-0006

43. Sun FL, Zhao G, Wang W (2015) Analysis method of igps measurement uncertainty spatial distribution. J Beijing Univ Aeronaut Astronaut 41(1):174-180

44. Tyrrell, M.: Machining a future (2020). https://www.aero-mag. com/amrc-machining-boeing-sheffield-220120/

45. VDI Verein Deutscher Ingenieure, VDE Verband der Elektrotechnik Elektronik Informationstechnik (2019) Inspection process management: Determination of the measurement uncertainty of complex inspection processes

46. Wasserstein RL, Lazar NA (2016) The asa statement on p -values: context, process, and purpose. Am Stat 70(2):129-133. https://doi. org/10.1080/00031305.2016.1154108

47. Welch BJ (1947) The generalisation of student's problems when several different population variances are involved. Biometrika 34(1-2):28-35. https://doi.org/10.1093/biomet/34.1-2.28 